\title{
Errata: Simultaneous photoacoustic imaging technique using an acoustic imaging lens
}

\author{
Xian Chen, ${ }^{a}$ Zhilie Tang, ${ }^{a, b, c}$ Yongheng He, ${ }^{a}$ Haifeng Liu, ${ }^{a}$ \\ Yadong Wei, ${ }^{a}$ and Yongbo $\mathrm{Wu}^{\mathrm{a}}$ \\ ${ }^{a}$ South China Normal University, School of Physics and \\ Telecom Engineering, 510006, Guangzhou, China \\ ${ }^{b}$ South China Normal University, Laboratory of Quantum \\ Information Technology, IMOT, 510006, Guangzhou, \\ China \\ ${ }^{\mathrm{c}}$ South China Normal University, Laboratory of Photonic \\ Information Technology, 510006, Guangzhou, \\ China \\ [DOI: $10.1117 / 1.3223339]$
}

This article [J. Biomed. Opt. 14, 030511 (2009)] was originally published online on 26 June 2009 with an incorrect image for Fig. 3. The correct image appears below.

All online versions of the article were corrected on 9 July 2009 and the article appears correctly in print.

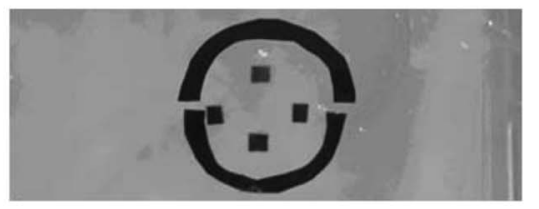

(a)

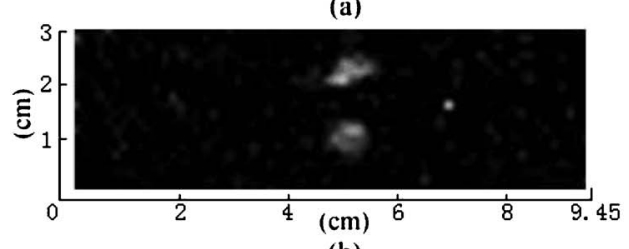

(b)

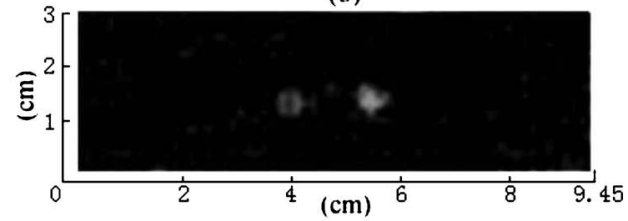

(c)
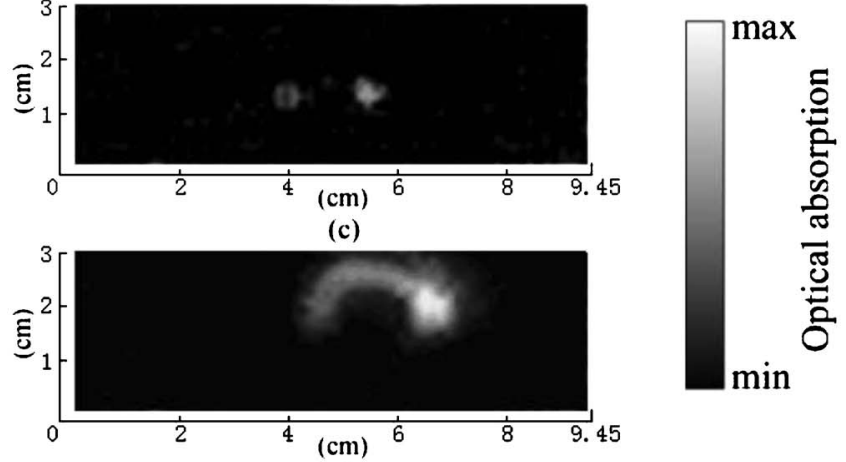

(d)

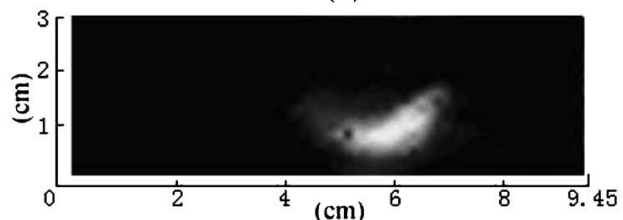

(e)

Fig. 3 Front elevation of (a) the sample, which consists of four different patterns: two lengthways black adhesive tape dots stuck to the front, two transverse black adhesive tape dots stuck to the second layer, a lower half ring of black adhesive tape stuck to the third layer, and an upper half ring of black adhesive tape stuck to the back of three pieces of polymethylmethacrylate. (b-e) are the corresponding reconstructed PA images of the four parts of the sample. 